\title{
Parafoveal-on-foveal effects are not an artifact of mis-located saccades
}

\author{
Alan Kennedy \\ School of Psychology, University of Dundee \\ and \\ Laboratoire de Psychologie et Neurosciences Cognitives, \\ Université René Descartes, Paris.
}

\begin{abstract}
Oculomotor error leads to a proportion of saccades during reading missing their intended target. Two kinds of mislocation occur: either a word is erroneously re-fixated, or a word that was about to skipped is erroneously fixated. In both cases recorded fixation duration could be influenced by the fact that the overt fixation reflects neither the reader's intentions, not the current locus of attention. It has been argued that mislocations of this kind account for apparent "parafoveal-on-foveal" interactions and that, consequently, the challenge posed by such effects for serial processing models of eye movement control is more apparent than real. It is argued here that this analysis is flawed: mislocated fixations cannot plausibly act within the architecture of a serial model to produce effects mimicking parafoveal-on-foveal cross-talk. The claim that parafoveal-on-foveal effects are restricted to measurements made when the eyes are very close to the relevant parafoveal target is not supported in an analysis of the effects of cumulative lexical frequency on foveal processing time.
\end{abstract}

Keywords: Oculomotor error, fixation duration, foveal processing time, mislocated saccades, Parafoveal-on-foveal effects

\section{Introduction}

The term "parafoveal-on-foveal effect" refers to the possibility that properties of a word present in the parafovea (i.e not directly inspected), may influence the way a currently fixated word is processed. Early analyses of this form of cross-talk (Kennedy, 1995; 1998) examined word pairs presented in isolation under laboratory conditions. The results showed that length, lexical constraint and frequency of an adjacent word in parafoveal vision all modulated foveal processing time. This pattern of findings was later reproduced in more complex tasks, involving short strings of words (Kennedy, 2000; Kennedy, Pynte \& Ducrot, 2002) and short sentences (Pynte, Kennedy \& Ducrot, 2004). In subsequent studies the range of parafoveal properties that appeared to influence foveal processing was extended to include the pragmatic plausibility of short sentences (Murray \& Rowan, 1998; Kennedy, Murray \& Boissiere, 2004). Taken together, these data obviously call into question the assumption that eye movement control in reading is an essentially serial activity in which "word objects" are processed one by one (McConkie, 1979; Morrison, 1984). Parafoveal-on-foveal effects cannot be accounted for in either a single-stage model of eye movement control based on the serial archi- tecture initially proposed by Morrison (see also Henderson \& Ferreira, 1993) or more complex two-stage models like E-Z Reader (Reichle, Pollatsek, Fisher \& Rayner, 1998; Reichle, Rayner \& Pollatsek, 2003) in which control over eye movements is decoupled from control over the allocation of covert attention. It is true that this decoupling provides opportunities for a degree of parallel processing (e.g. involving early visual processing), but parallel access to lexical constraint, word frequency or pragmatic plausibility cannot occur. Even if some early "familiarity check" at the point of fixation releases an eye movement, the specification of its target will be a function of the duration of the current fixation. Thus, evidence that the supra-lexical, lexical and some sub-lexical properties of an as-yet-un-fixated word might influence processing efficiency on a currently-fixated word is simply incompatible with the operation of a discrete attentional switch.

In this theoretical context it is hardly surprising that early demonstrations of parafoveal-on-foveal effects were followed by a number of studies querying their reliability (see Rayner \& Juhasz, 2004, for a brief review). The consistency, direction and stability of effects were called into question (Hyönä \& Bertram, 2004) and there were reports 
of failures to replicate (Inhoff, Radach, Starr \& Greenberg, 2000; Rayner, Warren, Juhasz, \& Liversedge, 2004). It is possible that some of these apparent inconsistencies arose from a failure to control relevant variables, in particular the length (and hence the visibility) of adjacent words (Kennedy et al., 2002; Kennedy \& Pynte, 2005). But even if the effects were genuine, it remained the case they appeared restricted to rather unusual presentation conditions, possibly not typical of normal reading. Specific task demands can affect eye movement control (Rayner \& Fischer, 1996) and it is possible that such strategies might emerge in rather artificial laboratory tasks. This was the position adopted by advocates of serial processing models until reliable parafoveal-on-foveal effects were found in two large-scale corpus analyses (Kennedy \& Pynte, 2005; Kliegl, Nuthmann \& Engbert, 2006). Since in each case the relevant corpus was based on eye movements recorded during the normal reading of extended text, the proposal that the effects resulted from task-specific strategies was no longer sustainable. Kliegl, Nuthmann and Engbert argue that their data are more compatible with an essentially parallel processing model (SWIFT) in which lexical properties are available across the visual span from typically three (but possibly more) words. Kennedy and Pynte also argue for parallel access to properties from at least two adjacent words, with eye movement control adjusted to maximize the visibility of target words.

Faced with these results, the response from advocates of the serial approach has taken both an empirical and a theoretical form. Rayner, Juhasz \& Brown (2007) point out that models like SWIFT, postulating a gradient of attention, must predict lexical parafoveal effects measured on Word $\mathrm{N}$ must be driven not only from the next Word $\mathrm{N}+1$, but also from Word $\mathrm{N}+2$. In an ingenious manipulation, Rayner et al. (2007) set a contingent boundary at the left edge of either Word $\mathrm{N}$ or Word $\mathrm{N}+1$. A strictly serial model predicts no preview effect from Word $\mathrm{N}+2$ and this was the result found. The outcome is persuasive because a preview effect is more robust and larger than typical estimates of parafoveal-on-foveal effects ${ }^{1}$. Nonetheless, the outcome has not gone unchallenged: Kliegl, Risse and Laubrock, (2007) replicated the results with regard to a lack of preview benefit on Word $\mathrm{N}+2$, but nonetheless found reliable parafoveal-on-foveal effects exerted from both Word $\mathrm{N}+1$ and Word $\mathrm{N}+2$.
The balance of empirical evidence suggests that measured effects of parafoveal word properties on foveal processing are genuine in the sense that they are not measurement artifacts, and are not restricted to tasks unrelated to normal reading. Nonetheless, from a theoretical perspective, it is rather unclear whether this conclusion forces an interpretation in favour of parallel processing. Several contrary lines of argument can be advanced. First, corpus analyses, while certainly informative, are by definition post hoc. Rayner, Pollatsek, Drieghe, Slattery and Reichle (2007) argue that retrospective statements of correlation can never be as informative as "true experiments" in which the properties of interest are manipulated factorially. There is some force to this argument, but it may be misplaced in the context of a discussion of linguistic or psycholinguistic properties that typically cannot be manipulated, in the sense that it is not possible, for example, to assign the same word to high and low levels of a factor like "Word Frequency". A further problematic aspect to the use of naturalistic texts is the fact that they contain proportionately many more short words than are found in typical psycholinguistic experiments and this may lead to biases. In particular, content and function words may exert different effects in parafoveal vision. Whether this argument is seen as convincing or not is bound to be affected by the fact that it is a reversal of the argument that parafoveal-on-foveal effects are restricted to "un-natural" laboratory tasks. But even if accepted, all that can be concluded is a claim the effects might be more complex than was initially thought: it is hardly a reason to conclude that they do not occur (see Pynte \& Kennedy, 2007 , for a discussion of the role punctuation might play in modulating cross-talk).

A far more convincing theoretical counter-argument rests in the claim that serial models like E-Z Reader, under appropriate circumstances, can accommodate, and even predict, apparent high-level parafoveal-on-foveal effects (Pollatsek, Reichle \& Rayner, 2006; Reichle, Pollatsek \& Rayner, 2006). The argument in this case rests on evidence of both systematic and random error in saccade control (McConkie, Kerr, Reddix \& Zola,1988; McConkie, Kerr, Reddix, Zola Jacobs, 1989). Saccadic error of this kind inevitably means that a number of saccades in normal reading probably lead to fixations of an unintended target. In favour of the serial approach it can be argued that mis-located fixations may give rise to proxy effects that mimic parafoveal cross-talk but are actually wholly artifactual. The present paper sets out to

\footnotetext{
${ }^{1}$ The study found no parafoveal-on-foveal effects either.
} 
examine this particular line of argument. Can a "mislocation" artifact account for obtained parafoveal-onfoveal effects in the context of the architecture of a serial model like $E-Z$ Reader?

\section{"Mis-location" and re-fixation}

There is little doubt that mis-location occurs. Measured landing positions during reading approximate a normal distribution, truncated at the word boundary (McConkie et al., 1988; 1989). The truncation relates to the fact that the missing tails of the distributions for any given word involve landings on adjacent words or the spaces between words. This pattern of under-shoot and over-shoot is usually ascribed to two sources: systematic range errors and oculomotor error. The term "range error" was used by Poulton (1981) to refer to choice biases induced by the knowledge of a range of possible targets. It seems unlikely that this sort of relatively high level cognitive control affects saccade targeting and, in fact, there is little evidence that true range effects influence eye movement control (Kapoula, 1985; Kapoula \& Robinson, 1986). For example, while saccadic under-shoots are often associated with remote targets in reading, systematic over-shoots to near targets do not occur (Vitu, 1991). On the other hand, the evidence that oculmotor noise influences saccade control is overwhelmingly strong. An important consequence for the argument to be developed here is that, depending on the degree of error, mis-located fixations may be associated with short-latency corrective saccades. This is because the landing position following a given saccade is monitored with respect to an efference copy of the relevant motor signal (see Nuthmann, Engbert \& Kliegl, 2005, for further discusssion) ${ }^{2}$. Mislocation artifacts of this kind have provided a convincing account of the counter-intuitive "Inverted Optimum Viewing Position Effect"; that is, the observation that fixation durations following a landing near the word centre tend to be longer than those landing near the word boundary $^{3}$ (Vitu, McConkie, Kerr \& O'Regan, 2001). This outcome appears less strange once it is appreciated that fixations remote from the Optimal Viewing Position may have been mis-locations followed by fast corrections
(Nuthmann, et al., 2005; but see Vitu, Lancelin \& d'Unienville, 2007, for an alternative account). Thus, mislocation artifacts may well account for one set of apparently paradoxical results. The question is, can they also account for parafoveal-on-foveal effects?

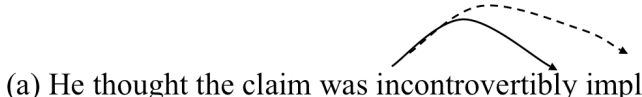

(a) He thought the claim was incontrovertibly implausible and

(b) He thought theories were often implausible and

Figure 1: (a) Mis-location leading to an unintended re-fixation; (b) mis-location following a failed skip leading to an erroneous single fixation. Dotted lines show the intended target and solid lines the actual saccade executed.

Figure 1a illustrates a single saccade launched from the word "incontrovertibly" towards the word "implausible". A "mis-location" artifact, considered within the twostage serial architecture of the E-Z Reader model would take the following form (Rayner et al, 2003; Pollatsek et al., 2006 Reichle, et al., 2006). Following fixation of the word "incontrovertibly", an initial stage of identification (L1) will be initiated. As soon as this initial process is satisfied (i.e. before word identification) a saccade is programmed towards the next word in the sentence (the word "implausible"). The dotted line illustrates the course of this intended saccade. When the fixated word "incontrovertibly" has been identified (i.e. stage L2 has

\footnotetext{
2 This is not the universally adopted position. Beauvillain and Vergilino (2000) for example, argue that some intra-word re-fixations are planned before initial fixation (see also Radach \& Heller, 2000). This does not allow advocates of the serial position off the hook, however, because the alternative to fast correction is inflated pre-skip duration.

${ }^{3}$ This appears paradoxical because the position where word identification should be easiest is apparently associated with longer, not shorter, processing time.
} 
been satisfied), attention will switch to the word "implausible" and processing of that word will begin although at that point in time it is not actually fixated. If oculomotor error intervenes in this process the actual saccade executed (solid line) may fall short of its target. The result is an unintended re-fixation. When this happens, in terms of the canonical serial model, the reader will be attending and processing the word "implausible" although actually fixating the word "incontrovertibly". Putting the matter in more general terms, data calibrated ${ }^{4}$ from this event will be assigned to Word $\mathrm{N}$ because that is where the eyes are located, but processing underway on Word $\mathrm{N}+1$ may influence the recorded data. It is important to note that both L1 and L2 for Word N must have been satisfied for this outcome to occur. This may not happen very often, but perhaps frequently enough to produce apparent parafoveal-on-foveal effects 5 .

Although this account is extremely ingenious, it has a number of shortcomings. The first problem is perhaps the most obvious. If mis-location leads to erroneous refixation, it is clearly predicted that parafoveal-on-foveal effects should not be found when the critical foveal word is processed with a single fixation. The data do not support this contention. Kennedy and Pynte (2005, Tables 1 and 2) show that parafoveal lexical frequency has a highly significant influence on single fixation duration measured on short foveal words. For obvious reasons single fixations on long foveal words were less common, but there too, there were significant parafoveal-on-foveal effects of both lexical frequency and "Informativeness". The Kennedy and Pynte analyses involved post-hoc allocation of stimuli to levels using median splits. The corpus analyses of Kliegl et al., (2006) used a more powerful repeated measures multiple regression technique and also showed clear parafoveal-on-foveal effects (in their terms, "successor" effects) in a measure of single fixation duration (with foveal length in the regression model). Thus, if mis-location necessarily leads to re-fixation, the data are in clear conflict with the predicted outcome.

A second problem relates to the fact that, at the time a mis-location occurs, Word $\mathrm{N}$ has been fully identified and attention turned to Word $\mathrm{N}+1$. It is necessary to consider how a serial model like $E-Z$ Reader would characterize such a situation. One possibility is that the (erroneous) refixation of Word $\mathrm{N}$ is treated as a landing on Word $\mathrm{N}+1$, albeit in an extremely bad position. The mechanism in the $E-Z$ Reader model for dealing with a poor initial landing position has changed over time, but involves a re-fixation to locate the eyes in a better position. As noted above, such a fast automatic response is pre-lexical. In which case, any resulting modulation to fixation duration on Word $\mathrm{N}$ cannot in principle relate to high-level properties of the attended Word $\mathrm{N}+1$. In this case the model no longer predicts parafoveal-on-foveal effects arising from mis-location. An alternative to the execution of a fast corrective saccade might be to remain in a poor position (i.e. fixating Word N), but continue to process the word that the system "believes" it is inspecting (Word N+1). This seems rather implausible. For example, in terms of Figure 1a it would involve the claim that the letter "l" at the end of the word "incontrovertibly" is somehow treated as belonging to the attended word "implausibly", a word that certainly contains the letter "l", but not in an initial position. But even setting considerations of plausibility aside, this interpretation leads to a counter-intuitive prediction. Eventually, the initial stages of identification on the attended "word object" will be satisfied, even from a non-optimal position, and a saccade programmed to the next word. But the question arises as to which the "next word" is? In processing terms it must be Word $\mathrm{N}+2$ (i.e. the word "and" in Figure 1a). That is, this "stay and process" alternative predicts that an erroneous re-fixation on Word $\mathrm{N}$ should be followed by a skip to Word $\mathrm{N}+2$. In the $E-Z$ Reader model, fixation duration prior to a skip is invariably inflated because a skip only occurs when a parafoveal word has been completely identified. Thus, artifactual parafoveal-on-foveal effects in this case should take the form of a global increase in processing time. Unfortunately, this prediction is also in conflict with the obtained data. In both laboratory studies (Kennedy, 2002) and corpus analyses, there are reports of reliable inverted effects of parafoveal lexical frequency. For example, a high frequency parafoveal target may lead to additional

\footnotetext{
${ }^{4}$ In some early critiques (e.g. Rayner et al., 2003) the idea was floated that poor eye tracker resolution might contribute to systematic error. But since any such error would have to shadow a rather complex pattern of interacting effects the proposition has been dropped.

${ }^{5}$ The debate could, of course, be ended at this point with the claim that is little reason to term such a parafoveal-on-foveal effect "apparent" rather than real.

${ }^{6}$ Informativeness was defined as the number of words sharing the target's initial three letters (Pynte, Kennedy \& Murray, 1991). It is important to note that this was measured independently of orthographic familiarity.
} 
foveal re-fixation, resulting in a paradoxically reversed "frequency effect". In summary, normal mis-locations within the architecture of the E-Z Reader model either lead to a corrective saccade that occur too soon to mimic parafoveal-on-foveal effects, or lead to skip-induced increases in processing time that are in conflict with the empirical data.

\section{Planned skips and failed skips}

There is an important exception to the claim that mislocation invariably leads to apparent re-fixation. This is illustrated in Figure 1b. The reader in this case planned to skip the word "theories" and execute a saccade directly to the word "were". In this case, oculomotor error leads to an erroneous landing on the word "theories" - a word the reader never intended looking at. More generally, a planned skip from Word $\mathrm{N}$ to Word $\mathrm{N}+2$ may fall short and land on Word $\mathrm{N}+1$, a word already completely identified and not intended to be fixated. Such an outcome could be relatively common, given that a short-fall is associated with longer saccades. Thus, a situation may arise where an unintended single fixation falls on a word already processed (Word $\mathrm{N}+1$ ) in circumstances where the following adjacent word $(\mathrm{N}+2)$ actually defines the attended target. Since a skip in the $E$ - $Z$ Reader model can only follow complete identification of the to-be-skipped word, the conditions for an artifactual parafoveal-onfoveal effect are satisfied. That is, Word $\mathrm{N}+1$ has been identified, but is erroneously fixated while Word $\mathrm{N}+2$ is attended and processed. Again, proxy effects driven by properties of the attended word might influence measures taken on an erroneously fixated word and these will appear to take the form of a parafoveal-on-foveal influence.

This "failed skip" artifact appears to offer better news for the strictly serial model, but in fact it must fail for reasons similar to those bearing on the "re-fixation artifact" already considered. Consider Figure 1b. It is assumed that the word "were" is being processed while the word "theories" is (erroneously) fixated. Consequently, when the initial stage in the identification of the word "were" is completed a saccade will be programmed to the word "often". Thus, the prediction is that a failed skip should be followed by a planned skip and any artifactual parafoveal-on-foveal effect will inevitably take the form of a skip-induced increase in processing time ${ }^{7}$. As noted above, the evidence does not appear to support this.

\section{"Wordness" as a parafoveal property}

Although the theoretical argument advanced above appears challenging for adherents of the serial model there has been at least one recent attempt to provide direct empirical support for the "mis-location" hypothesis. Drieghe Rayner and Pollatsek (2008) examined eye movements deployed over sequences of three words (e.g. young child performing) embedded in short sentences. The first word (a high frequency short word) provides a control for launch position. Properties of the second word ( e.g. child) were manipulated to be of either high or low frequency. Using the boundary condition (Rayner, 1975), on half the trials the legality of the orthography of the third word was changed when in parafoveal vision (e.g. performing was displayed as pxvforming). Drieghe et al. found an effect of parafoveal orthography on foveal single fixation time. That is, inspection time on the word child was inflated when the third word appeared in its orthographically illegal form. Crucially, however, this effect of parafoveal illegality only arose when the fixation on the foveal word fell on its final letter. When measured at all other positions there was no effect at all. The authors conclude, in the words of their title, that "mis-located fixations can account for parafoveal-onfoveal effects in eye movements during reading."

There are good reasons for caution in accepting this conclusion. First, it should be noted that the measure of foveal processing time employed was single fixation duration. Indeed, 96 percent of all cases fell into this category. Given the analysis above, it is certainly possible that an unknown proportion of these were, in fact, "mislocated" in the sense that the actual target was not the intended target. As noted above, where this occurred it must have resulted from a failed skip and the direction of the obtained effect (a relatively large inflation in inspection time) is consistent with this. However, the fact the effect was restricted to landings on the final letter is harder to explain. An effect resulting from oculomotor error should presumably have a normal distribution, with diminishing impact as distance from the parafoveal word boundary increases. A second problem relates to the fact

\footnotetext{
${ }^{7}$ It should be emphasized that this prediction may itself be in error. Kiegl \& Engbert (2005) suggest that fixation duration is reduced when the skipped word is short or of high frequency and increased when it is long or low frequency (ses also Pynte \& Kennedy, 2007).
} 
that the parafoveal manipulation concerned orthographic legality. It is unclear whether this property should be treated alongside the range of lexical and supra-lexical properties claimed to induce parafoveal-on-foveal effects. Orthographic illegality manifests itself as "oddity" or "strangeness" in parafovea vision, something that might be detectable in very early visual processing. Indeed, some versions of the E-Z Reader model have explicitly proposed that early processing in the visual system might connect directly to the labile stage (M1) in the oculomotor system. Thus, the contrast word vs non-word may provide a particularly good test-bed for examining lexical parafoveal-on-foveal effects. Pynte, Kennedy and Ducrot (2004) examined this question directly, comparing foveal processing in the vicinity of correctly-spelled or misspelled parafoveal targets differing in initial letter constraint. The materials were in French and contrasted words like "vrombir" (the only seven letter word in French beginning with the letters v,r,o) with "croupir" (a relatively unconstrained initial trigram). The boundary procedure was employed to ensure that mis-spelled (nonword) versions of these items ("crombir" and "vroupir") only appeared in the parafovea. This experiment differed in one important way from that conducted by Dreighe et al: the critical foveal word was always separated from the parafoveal target by a short preposition (e.g. "...commencer à vrombir..."). In this case, for example, any parafoveal-on-foveal effects measured on the word "commencer" must result from fixations at least three character positions away. The results showed pronounced effects of parafoveal constraint on foveal inspection time, with shorter times associated with highly contrained targets. On the other hand, there was no measurable effect of lexical status ("wordness") at all.

How can these two outcomes be reconciled? One possible route is to note the size of the effect obtained by Drieghe et al. Correct and incorrect preview conditions were almost identical at all fixation fixations, apart from the final letter. In that position, foveal single fixation duration was about $90 \mathrm{~ms}$ longer. The size of this effect is almost an order of magnitude greater than parafoveal-onfoveal effects typically attributed to lexical frequency, initial letter constraint, or orthographic familiarity. These fall into the 5 - $20 \mathrm{~ms}$ range and their demonstration demands relatively high power in an experimental design. Thus, one tentative conclusion is that the post-facto analysis by-launch position carried out was extremely unlikely to have sufficient power to demonstrate small effects. A second, and more important, conclusion is that the effect obtained, while striking, appears to relate to visual rather than lexical properties. This is of interest, but may not contribute to the on-going debate as to whether lexical and sub-lexical sources of control over eye movements in reading are better characterized as "serial" or "parallel".

\section{Exploring orthographic familiarity in the Dundee Corpus}

In this final section the role played by inspection position is assessed directly by means of an analysis of the English Dundee Corpus. As noted above, several reliable parafoveal-on-foveal effects have been reported based on analyses of this corpus. If the argument advanced by Drieghe et al. is correct it should be the case that these effects are restricted to cases where the relevant foveal fixation is located close to the parafoveal target (i.e. within a couple a characters).

The English part of the Dundee Corpus data comprises eye movements recorded from 10 English-speaking participants as they read text taken from editorials in The Independent newspaper. Texts comprising 56,212 tokens and 9,776 types in total, were presented on a screen five lines at a time, double-spaced, using a line length of 80 characters. The position of the right eye was sampled every millisecond, using a Dr Bouis Oculometer Eyetracker. Viewing distance was $500 \mathrm{~mm}$ and one character subtended approximately $0.3^{\circ}$ of visual angle. The data were collected over several days of testing. Inspection parameters were computed using statistical algorithms based on the resolution of the data for each individual participant with respect to the obtained noise in a given data set. The effective resolution of the eye-tracking system was considerably better than one character position (Kennedy, Hill \& Pynte, 2003; Kennedy \& Pynte, 2005).

It is not, of course, possible to examine the effects of orthographic illegality directly in such a corpus analysis, but a plausible surrogate measure is provided by the index of parafoveal lexical familiarity. This represents the "cumulative lexical frequency" for a given word; that is, for each word, the summed frequency of all words sharing that token's initial trigram. The measure provides a rough index of the visual familiarity of a given initial trigram (i.e. the overall likelihood of encountering it in text). It may be compared with orthographic legality at least in the sense that both are relatively "low-level" measures. Although cumulative lexical frequency in practice correlates highly with lexical frequency it is, in prin- 
ciple, capable of being dissociated from both frequency and initial letter "informativeness". Indeed, Kennedy and Pynte (2005) examined these three factors independently in an orthogonal design using a post-hoc allocation by means of median splits. However, this procedure is unsuited to the examination of the effects of fixation position because of the massive attrition in the available data sets. For this reason, the present analysis followed the procedure employed by Kliegl (2007) and used linear mixed-effects analyses (Pinheiro \& Bates, 2000) conducted over a substantial sub-set of the Corpus as a whole. The lme4 package (Bates \& Sarkar, 2006) in the R system for statistical computing was used (R Development Core Team, 2006; see also Baayen, 2007 and Venables \& Smith, 2002) to carry out repeated measures multiple regression analyses. The dependent variable was foveal single fixation duration. Participants and Items were treated as random factors. There were two fixed effects: measured parafoveal Familarity (i.e. cumulative lexical frequency) and "Informativeness" (initial letter constraint, defined as the number of words of similar length sharing the initial three letters). All words between 5 and 20 characters in length were entered.

An initial exploratory analysis was carried out with an additional fixed effect defined as the distance in characters between a given foveal fixation and the left boundary of the parafoveal target. There were two significant effects: Parafoveal Familiarity $(\mathrm{B}=-2.29$, S.E. $=0.84, \mathrm{t}=$ -2.73, 36312 observations) and Launch Position ( $\mathrm{B}=$ 5.04, S.E. $=0.83, \mathrm{t}=6.10)$. Overall, the more "familiar" the (unfixated) parafoveal stimulus the shorter the single fixation on the prior foveal word. Since Launch Position was defined as negative values (from -1 to -9 characters), its effect can be interpreted as showing an increase in foveal fixation duration, the closer the eyes were to the foveal target. No other effects and no interactions were significant (all $\mathrm{t}<1$ ); in particular, the interaction between Familiarity and Launch Position was not significant. Clearly, there is a reliable parafoveal-on-foveal effects of target Familiarity ${ }^{8}$; but the critical question is whether this is all, or largely, contributed by possible mislocations - cases when the eyes were very close to the target. Although the failure to demonstrate a significant interaction suggests a negative answer, the question can be addressed directly by means of separate analyses conducted for fixations spanning the range from -1 character (the critical point discussed by Drieghe et al.) to -9 characters. Table 1 shows regression coefficients, standard errors and t-values for these cases, together with an indication of the number of observations recruited to each analysis.

Table 1

Regression coefficients, standard errors and t-values for the fixed effect Parafoveal Cumulative Lexical Frequency.

\begin{tabular}{ccccc}
\hline $\begin{array}{c}\text { Relative } \\
\text { Position }\end{array}$ & B & S.E. & $\mathrm{t}$ & No Obs \\
\hline-1 & -1.47 & 1.54 & -0.95 & 3268 \\
-2 & -2.47 & 1.09 & -2.26 & 4501 \\
-3 & -2.12 & 1.07 & -1.98 & 5435 \\
-4 & -0.81 & 0.84 & -0.96 & 5917 \\
-5 & -2.81 & 0.84 & -3.36 & 5572 \\
-6 & -2.45 & 0.78 & -3.13 & 5025 \\
-7 & -2.67 & 1.16 & -2.3 & 3293 \\
-8 & 0.16 & 1.42 & +0.11 & 2094 \\
-9 & 1.6 & 1.69 & +0.95 & 1207 \\
\hline
\end{tabular}

Note. The Dependent Variable is foveal Single Fixation Duration. The data are shown as a function of the position of the foveal fixation relative to its parafoveal target (the inter-word space being -1).

Two conclusions can be drawn from this Table. First, the majority of cases fall in the range -3 to -5 characters: fewer fixations are located a single character from the target and, equally, it is relatively unusual for a single fixation to be located 8 or 9 characters from the target. The second point is that (with one exception) there is a significant effect of target Familiarity across the range from -2 to -7 characters. There is no effect when the fixation fell on the inter-word space and no effect when the foveal fixation was relatively remote. As noted above, these analysis do not address the issue of parafoveal "wordness" directly, but they do show that at least one low-level parafoveal property has an effect on foveal processing that is not restricted to very close launch positions.

\footnotetext{
${ }^{8}$ Since the two measures correlate highly it was not prudent to include parafoveal word frequency as a further fixed effect. However, in the context of the present debate, since a parafoveal-on-foveal frequency effect would be construed as a "high-level", the present interpretation in terms of visual "familiarity" is theoretically conservative.
} 


\section{Conclusion}

Mis-located fixations occur in reading, but their effects are unlikely to be systematic. They are an inescapable source of random noise, affecting measured fixation location and duration. However, it is implausible they lead to a pattern of artifact shadowing the range of obtained parafoveal-on-foveal effects. It is more likely that such effects are genuine and reflect overlapping processes in which properties from adjacent words, at all levels, are available in parallel.

\section{Acknowledgements}

This research was supported by a grant from the UK Economic and Social Research Council.

\section{References}

Bates, D., \& Sarkar, D. (2006). lme4: Linear mixed-effect models using 54 classes. R package version 0.995-2.

Baayen, R.H. (2007). Analyzing linguistic data: A practical introduction to statistics. Cambridge: Cambridge University Press.

Drieghe, D., Rayner, K. \& Pollatsek, A. (2008). Mislocated fixations can account for parafoveal-on-foveal effects in eye movements during reading. Quarterly Journal of Experimental Psychology.

Henderson, J. M. \& Ferreira, F. (1993) Eye movement control during reading: Fixation measures foveal but not parafoveal processing difficulty. Canadian Journal of Experimental Psychology 47:201-21.

Hyönä, J., \& Bertram, R. (2004). Do frequency characteristics of nonfixated words influence the processing of fixated words during reading? European Journal of Cognitive Psychology, 16, 104-127.

Inhoff, A.W., Radach, R., Starr, M., \& Greenberg, S. (2000). Allocation of visuospatial attention and saccade programming during reading. In A. Kennedy, R. Radach, D. Heller, and J. Pynte (Eds.). Reading as a Perceptual Process. Pp. 221-246). Amsterdam: Elsevier.

Kapoula Z (1985) Evidence for a range effect in the saccadic system. Vision Research, 25, 1155-1157.

Kapoula Z. \& Robinson DA. (1986) Saccadic undershoot is not inevitable: saccades can be accurate. Vision Research, 26, 735-743.
Kennedy, A. (1995).The influence of parafoveal words on foveal inspection time. AMLaP-95 Conference, Edinburgh, 1995.

Kennedy, A. (1998). The influence of parafoveal words on foveal inspection time: evidence for a processing trade-off. In G. Underwood (Ed.). Eye Guidance in Rreading and Scene Perception (pp 149 - 223). Oxford: Elsevier.

Kennedy, A. (2000a). Parafoveal processing in word recognition. Quarterly Journal of Experimental Psychology, 53A, 429 - 455.

Kennedy, A., \& Pynte, J. (2005). Parafoveal-on-foveal effects in normal reading. Vision Research, 45, 153168.

Kennedy, A., Hill, R., \& Pynte, J. (2003). The Dundee corpus. Poster presented at ECEM12: 12th European Conference on eye movements., Dundee, August 2003.

Kennedy, A., Murray, W. S. \& Boissiere. (2004). Parafoveal Pragmatics revisited. European Journal of Cognitive Psychology, 16, 128-153.

Kennedy, A., Pynte, J., \& Ducrot, S. (2002). Parafovealon-foveal interactions in word recognition. Quarterly Journal of Experimental Psychology, 55A, 1307-1337.

Kliegl, R. (2007). Towards a perceptual-span theory of distributed processing in reading: A reply to Rayner, Pollatsek, Drueghe, Slattery \& Reichle (2007). Journal of Experimental Psychology: General.

Kliegl, R., \& Engbert, R. (2005). Fixation durations before word skipping in reading eye movements. Psychonomic Bulletin \& Review. 12, 132-138.

Kliegl, R., Nuthmann, A., \& Engbert, R. (2006). Tracking the mind during reading: The influence of past, present, and future words on fixation durations. Journal of Experimental Psychology: General, 135, 12-35.

Kliegl, R., Risse, S., \& Laubrock, J. (2007). Preview benefit and parafoveal-on-foveal effects from wordn+2. Journal of Experimental Psychology: General.

McConkie, G. W. (1979). On the role and control of eye movements in reading. In P. A. Kolers, M. E. Wrolstad \& H. Bouma (Eds.). Processing of Visible Language: Vol. I (pp 37-48). New York: Plenum Press.

McConkie, G.W., Kerr, P.W., Reddix, M.D. \& Zola, D. (1988). Eye movement control during reading: I. The location of initial fixations on words. Vision Research, 28,11071118 . 
McConkie, G.W., Kerr, P.W., Reddix, M.D., Zola, D. \& Jacobs, A.M. (1989). Eye movement control during reading: II. Frequency of refixating a word. Perception and Psychophysics, 46, 245-253.

Morrison, R.E. (1984). Manipulation of stimulus onset delay in reading: Evidence for parallel programming of saccades. Journal of Experimental Psychology: Human Perception and Performance, 10, 667-682.

Murray, W. S. \& Rowan, M. (1998). Early, mandatory, pragmatic processing. Journal of Psycholingusitic Research, 27, 1 - 22.

Nuthmann, A., Engbert, R., \& Kliegl, R. (2005). Mislocated fixations during reading and the inverted optimal viewing position effect. Vision Research, 45, 2201-2217.

Pinheiro, J., \& Bates, D. (2000). Mixed-effects models in $S$ and S-Plus. New York: Springer.

Pollatsek, A., Reichle, E.D., \& Rayner, K. (2006). Tests of the E-Z Reader model: Exploring the interface between cognition and eye-movement control. Cognitive Psychology, 52, 1-56.

Poulton, E.C. (1981). Human manual control. In V.B. Brooks (Ed.). Handbook of physiology. Section 1: The nervous system. Volume II: Motor control. Part 2 (pp 13371389). Bethesda, Maryland: American Physiological Society.

Pynte, J. \& Kennedy, A. (2007). An influence over eye movements in reading exerted from beyond the level of the word: Evidence from reading English and French. Vision Research, 46, 3786-3801.

Pynte, J., Kennedy, A. \& Murray, W. S. (1991). Withinword inspection strategies in continuous reading: Time course of perceptual, lexical and contextual processes. Journal of Experimental Psychology: $\mathrm{Hu}$ man Perception and Performance, 17, 458-470.

Pynte, J., Kennedy, A., \& Ducrot, S. (2004). The influence of parafoveal typographical errors on eye movements in reading. European Journal of Cognitive Psychology, 16, 178-202.

R Development Core Team (2006). R: A language and environment for statistical computing. (version 2.3.1). R Foundation for Statistical Computing, Vienna, Austria.

Radach, R. \& Heller, D. (2000). Relations between spatial and temporal aspects of eye movements control. In A. Kennedy, R. Radach, D. Heller, and J. Pynte (Eds.). Reading as a Perceptual Process. (pp 165191). Amsterdam: Elsevier.
Rayner, K. (1975). The perceptual span and peripheral cues in reading. Cognitive Psychology, 7, $65-81$.

Rayner, K. \& Fischer, M.H. (1996). Mindless reading revisited: Eye movements during reading and scanning are different. Perception and Psychophysics, 58, $734-747$.

Rayner, K., \& Juhasz, B.J. (2004). Eye movements in reading: Old questions and new directions. European Journal of Cognitive Psychology, 16, 340352.

Rayner, K., Juhasz, B.J., \& Brown, S.J. (2007). Do readers obtain preview benefit from word $n+2$ ? A test of serial attention shift vs. distributed lexical processing models of eye movement control in reading. Journal of Experimental Psychology: Human Perception and Performance.

Rayner, K., Pollatsek, A., Drieghe, D., Slattery, T.J., \& Reichle, E.D. (2007). Tracking the mind during reading via eye movements: Comments on Kliegl, Nuthmann, and Engbert (2006). Journal of Experimental Psychology: General, 134,In Press.

Rayner, K., Warren, T, Juhasz, B.J., \& Liversedge, S.P. (2004). Eye movements and plausibility effects in reading. Journal of Experimental Psychology: Learning, Memory, and Cognition, 30, 1290-1301.

Rayner, K., White, S., Kambe, G., Miller, B. \& Liversedge, S. (2003). On the processing of meaning from parafoveal vision during eye fixations in reading. In J. Hyona, D. Munoz, W. Heide \& R. Radach (Eds.), The Mind's Eye: Cognitive and Applied aspects of Eye Movements. Oxford: Elsevier Science.

Reichle, E. D., Pollatsek, A., Fisher, D. L. \& Rayner, K. (1998). Towards a model of eye movement control in reading. Psychological Review, 105, 125-157.

Reichle, E. D., Rayner, K., \& Pollatsek, A. (2003). The E$Z$ reader model of eye movement control in reading: comparisons to other models. Behavioral and Brain Sciences, 26, 445-476.

Reichle,E.D., Pollatsek,A., \& Rayner,K. (2006). E-Z Reader: A cognitive-control, serial-attention model of eye-movement behavior during reading. Cognitive Systems Research, 7, 4-22.

Vergilino, D. \& Beauvillain, C. (2000). The planning of re-fixation saccades in reading. Vision Research, 40, 3527-3538.

Vitu, F. (1991). The existence of a center of gravity effect during reading. Vision Research, 31,1289-1313. 
Vitu, F., Lancelin, D. \& d'Unienville, V. M. (2007). A perceptual economy acount of the Inverted Optimal Viewing Position effect. Journal of Experimental Psychology: Human Perception \& Performance.

Vitu, F., McConkie, G. W., Kerr, P., \& O’Regan, J. K. (2001). Fixation location effects on fixation durations during reading: an inverted optimal viewing position effect. Vision Research, 41, 3513-3533. 